\title{
Combined defects in epithelial and immunoregulatory factors exacerbate the pathogenesis of inflammation: mucin 2-interleukin 10-deficient mice
}

\author{
Maria van der Sluis ${ }^{1}$, Janneke Bouma ${ }^{1}$, Audrey Vincent ${ }^{2}$, Anna Velcich ${ }^{3}$, Kermit L Carraway ${ }^{4}$, Hans A Büller ${ }^{5}$, \\ Alexandra WC Einerhand ${ }^{5}$, Johannes B van Goudoever ${ }^{1}$, Isabelle Van Seuningen ${ }^{2}$ and Ingrid B Renes ${ }^{1}$
}

Expression of the mucin MUC2, the structural component of the colonic mucus layer, is lowered in ulcerative colitis.

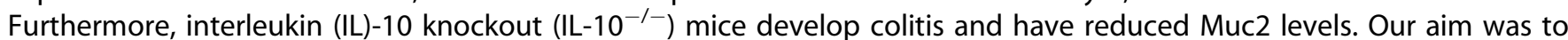
obtain insight into the role of Muc2 and IL-10 in epithelial protection. Muc2-IL-10 double-knockout (Muc2/IL-10 ${ }^{\mathrm{DKO}}$ ) mice were characterized and compared to Muc2 knockout $\left(\mathrm{Muc2}^{-/-}\right), \mathrm{IL}-10^{-/-}$and wild-type (WT) mice. Clinical symptoms, intestinal morphology and differences in epithelial-specific protein levels were analyzed. In addition, levels of the pro-

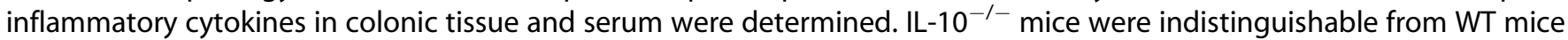
throughout this experiment and showed no clinical or histological signs of colitis. Muc2/IL-10 ${ }^{\mathrm{DKO}}$ and Muc2 ${ }^{-1-}$ mice showed significant growth retardation and clinical signs of colitis at 4 and 5 weeks, respectively. Muc2/IL-10 ${ }^{\mathrm{DKO}}$ mice had a high mortality rate ( $50 \%$ survival/ 5 weeks) compared to the other types of mice ( $100 \%$ survival). Microscopic analysis of the colon of Muc2/IL-10 ${ }^{\mathrm{DKO}}$ mice showed mucosal thickening, increased proliferation, superficial erosions and a diminished Muc4 expression. Furthermore, pro-inflammatory cytokines were significantly upregulated, both in tissue (mRNA) and systemically in Muc2/IL-10 ${ }^{\mathrm{DKO}}$ mice. In conclusion, Muc2/IL-10 $0^{\mathrm{DKO}}$ mice develop colitis, which is more severe in every aspect compared to Muc2 ${ }^{-1-}$ and IL-10 $10^{-1-}$ mice. These data indicate that (i) in case of Muc2 deficiency, the antiinflammatory cytokine IL-10 can control epithelial damage, though to a limited extent and (ii) the mucus layer is most likely a key factor determining colitis.

Laboratory Investigation (2008) 88, 634-642; doi:10.1038/labinvest.2008.28; published online 21 April 2008

KEYWORDS: experimental colitis; goblet cell; inflammatory bowel diseases; intestine; mice; mucin

Inflammatory bowel diseases (IBD), including ulcerative colitis (UC) and Crohn's disease, are characterized by chronic inflammation and mucosal tissue damage of parts of the gastrointestinal tract. They are thought to be caused by combined genetic, environmental, immunoregulatory and epithelial factors. ${ }^{1,2}$

Interleukin (IL)-10 contributes to maintaining intestinal homeostasis by suppressing pro-inflammatory cytokine production. ${ }^{1,3,4}$ IL-10-deficient (IL-10 ${ }^{-/-}$) mice spontaneously develop chronic colitis, of which severity is dependent on both strain background and environmental factors. ${ }^{4-6}$ Relocation of IL- $10^{-1-}$ mice from specific pathogen-free, conventional environments into germ-free isolators eliminates immune system activation and colitis development. ${ }^{7,8}$

The intestinal epithelium and mucus layer protect underlying tissues from potentially noxious agents in the lumen. The mucus layer is composed of mucins and one secretory mucin in particular, Muc2, is abundantly expressed in human, rat and mouse colonic epithelium..$^{9-13}$ We recently found that intestinal loss of Muc2, affecting the protective capacities of the mucus layer, leads to colonic inflammation in mice. ${ }^{14}$ Furthermore, the activity of mucosal inflammation in human UC correlates significantly with lowered MUC2 synthesis and secretion. ${ }^{15,16}$

'Division of Neonatology, Department of Pediatrics, Erasmus MC and Sophia Children's Hospital, Rotterdam, The Netherlands; ${ }^{2}$ Inserm, U837, Centre de Recherche Jean-Pierre Aubert, Lille, France; ${ }^{3}$ Oncology Department, Albert Einstein Cancer Centre/Montefiore Medical Centre, New York, NY, USA; ${ }^{4}$ Department of Cell Biology and Anatomy, University of Miami School of Medicine, Miami, FL, USA and ${ }^{5}$ Division of Pediatric Gastroenterology and Nutrition, Department of Pediatrics, Erasmus MC and Sophia Children's Hospital, Rotterdam, The Netherlands

Correspondence: Dr IB Renes, PhD, Division of Neonatology, Department of Pediatrics, Erasmus MC-Sophia Children's Hospital, Rm. Ee 15.75A, Dr Molewaterplein 50, Rotterdam 3015 GE, The Netherlands. E-mail: i.renes@erasmusmc.nl 
In the present study, we investigated the intestinal protective capacities of a Muc2/IL-10 double-knockout (Muc2/ IL-10 ${ }^{\text {DKO }}$ ) mouse model, determining whether combined defects in the immunoregulatory and epithelial factors could exacerbate the pathogenesis of inflammation. To this aim, we compared clinical symptoms and intestinal pathology of these mice to those matched Muc2 ${ }^{-1-}$ and IL- $10^{-1-}$ mice. By doing so, this animal model is the first to provide insight into contributions of both immunological and epithelial abnormalities, that is IL-10 and Muc2 deficiency, to the severity of colonic inflammation.

\section{MATERIALS AND METHODS Animals}

The previously described heterozygous Muc2-129SV mice and IL-10 $0^{-1-}$-BL6 mice (a kind gift from Dr M Mähler, Hanover, Germany), designated $I L-10^{\operatorname{tm} 1 C g n}$ were bred to generate heterozygous double-knockout mice. ${ }^{6,14,17}$ These were then bred with each other to generate homozygous Muc2/IL-10 ${ }^{\text {DKO }}$ mice.

Throughout the crossing procedure, the targeted Muc2 and IL-10 genes were monitored via PCR assays performed on genomic DNA isolated from tail clips. ${ }^{6,18}$ The mice were housed in the same specific pathogen-free environment, and all animal care and procedures complied with institutional guidelines (Erasmus MC-Animal Ethics Committee, Rotterdam, The Netherlands).

\section{Experimental Setup}

The initial setup of this experiment was to characterize wildtype (WT) $(n=24), \mathrm{IL}-10^{-/-}(n=20)$ and Muc $2 / \mathrm{IL}-10^{\mathrm{DKO}}$ $(n=23)$ littermates from 5 weeks until 16 weeks of age, consistent with our study on the Muc2 ${ }^{-1-}$ mouse. ${ }^{14}$ Adaptation proved necessary as the Muc2/IL- $10^{\mathrm{DKO}}$ showed high mortality rate and disease activity index (DAI). Muc2/ IL- $10^{\text {DKO }}$ and corresponding WT littermates were now sacrificed after 4 (Muc2/IL-10 ${ }^{\mathrm{DKO}}, n=5$; WT, $\left.n=4\right), 5$ (Muc2/ $\mathrm{IL}-10^{\mathrm{DKO}}, n=7$; WT, $\left.n=4\right)$ and 7 (Muc2/IL- $10^{\mathrm{DKO}}, n=1$ ) weeks. Controls, $\mathrm{IL}-10^{-1-}$ and corresponding WT mice, were sacrificed at $5,8,12,16$ and 34 weeks of age $(n=4$, each time point) as these develop colitis at a later age. ${ }^{7,19}$ Furthermore, findings in Muc2/IL-10 ${ }^{\mathrm{DKO}}$ mice were compared to our previously published findings in the Muc2 ${ }^{-/-}$mouse. ${ }^{14}$

Disease activity index was scored as described by Cooper et al $^{20}$ (Table 1). Epithelial-cell proliferation was determined by in vivo DNA labeling with bromodeoxyuridine (BrdU), injected intraperitoneally $30 \mathrm{mg} / \mathrm{kg}$ body weight (Sigma, St Louis, MO, USA) $1 \mathrm{~h}$ prior to killing. ${ }^{21,22}$ Small intestine and colon were excised and either fixed in $4 \%(\mathrm{w} / \mathrm{v})$ paraformaldehyde in PBS, stored in RNA later (Qiagen, Venlo, The Netherlands) at $-20^{\circ} \mathrm{C}$ or frozen in liquid nitrogen and stored at $-80^{\circ} \mathrm{C}$.

\section{Histology and Histological Grading}

Tissue fixed in $4 \%(\mathrm{w} / \mathrm{v})$ paraformaldehyde in PBS was prepared for light microscopy and $5-\mu \mathrm{m}$ thick sections were
Table 1 Disease activity index (DAI) score

\begin{tabular}{lllll}
\hline Score & $\begin{array}{c}\% \text { Weight } \\
\text { loss }^{\mathrm{a}}\end{array}$ & Stool consistency & Blood loss & Appearance \\
\hline 0 & None & Normal droppings & None & Lively/normal \\
1 & $0-25$ & Loose droppings & $\begin{array}{l}\text { Hemoccult- } \\
\text { positive }\end{array}$ & Hunched \\
& & & Gross & Starey coat \\
2 & $25-50$ & Diarrhea & bleeding & \\
3 & & & & Lethargic
\end{tabular}

${ }^{a}$ Criteria were obtained by pooling all data and calculating quartiles. The percentage weight loss was calculated by comparing the weight of the mice to their corresponding wild-type littermates.

stained either by (1) hematoxylin and eosin or (2) Alcian blue $(\mathrm{AB})$ at $\mathrm{pH} 2.5$ followed by periodic acid/Schiff's reagent (PAS) as described previously. ${ }^{23}$ Intestinal inflammation was characterized by number of goblet cells, mucosal thickening, presence of inflammatory cells, general destruction of architecture and presence of ulcers and/or crypt abscesses. All tissue samples were evaluated in a blinded fashion by two independent investigators.

\section{Immunohistochemistry}

Five-micrometer thick sections were cut and prepared for immunohistochemistry. ${ }^{22}$ Expression of Muc2 was detected by anti-Muc2 antibody H-300, (1:1000 diluted in PBS; SC15334, Santa Cruz, SanverTech, Heerhugowaard, The Netherlands). To detect goblet cells, anti-trefoil factor 3 (Tff3; 1:3000 diluted in PBS; a generous gift from Professor Dr DK Podolsky) was used. ${ }^{24}$ Muc4 was stained using anti-hMUC4 polyclonal antibody (hHA-1) that recognizes a C-terminal peptidic region of MUC4 $\alpha$ subunit (1:6000 diluted in 1\% BSA, $0.1 \%$ Triton X-100 in PBS). ${ }^{25}$ To visualize BrdU incorporation, sections were incubated with anti-BrdU (1:250 diluted in PBS; Boehringer Mannheim, Mannheim, Germany). ${ }^{26} \mathrm{CD}^{+}$cells were detected using an anti-human CD3 (1:800 diluted in 1\% BSA, $0.1 \%$ Triton X-100 in PBS; A0452, DAKO, Heverlee, Belgium). ${ }^{14}$

\section{Quantitative Real-Time PCR}

mRNA expression levels of the mucins Muc1, -3 and -4 , as well as the endogenous housekeeping gene $\beta$-actin were quantified using real-time PCR analysis based upon the intercalation of SYBR ${ }^{\circledR}$ Green on an ABI Prism 7700 sequence detection system (PE Applied Biosystems). ${ }^{14}$ The primer combinations for Muc1 (5'-CTTTCAGAAGACTCCGCCAG-3' and $5^{\prime}$-GGCCAAGACTGATTCAGAGC- $\left.3^{\prime}\right)$, Muc3 ( $3^{\prime}$-GAG ACATGCAAGAAGGAGGC- $5^{\prime}$ and $3^{\prime}$-CCAAGTCCATACA CCAGGCT- $5^{\prime}$ ) and Muc4 (5'-CCCGCTCATCCACTATC- $3^{\prime}$ and $5^{\prime}$-TGGCCTCCATTGTGAC- $3^{\prime}$ ) were designed using the 
OLIGO 6.22 software (Molecular Biology Insights, Cascade, CO, USA) and purchased from Invitrogen (Breda, The Netherlands). In addition, mRNA expression levels of tumor necrosis factor alpha (TNF- $\alpha$ ), IL- $1 \beta$ and IL- 6 as well as $\beta$-actin were quantified. ${ }^{14}$

\section{Serum Cytokine Levels}

Serum was obtained from coagulated blood collected by heart puncture $\left(15 \mathrm{~min}\right.$ at $37^{\circ} \mathrm{C}$, then $30 \mathrm{~min}$ a $\left.4^{\circ} \mathrm{C}\right)$ and stored at $-20^{\circ} \mathrm{C}$ until further analysis. The concentrations of several cytokines (IL-12, TNF- $\alpha$, interferon gamma (IFN- $\gamma$ ), monocyte chemoattractant protein-1 (MCP-1), IL-10 and IL-6) in serum were determined with a BD Cytometric Bead Array mouse inflammation kit (BD-Pharmingen, San Diego, CA, USA).

\section{Statistical Analysis}

All data are expressed as mean \pm s.e.m. Statistical significant differences between the three different types of mice over time were assessed by the one-way ANOVA test followed by a Tukey $T$-test. The Mann-Whitney $U$-test was used to analyze changes between two groups (PRISM, version 4.00; Graphpad software, San Diego, CA, USA). Differences were considered statistically significant at $P<0.05$.

\section{RESULTS}

\section{Clinical Symptoms}

Numbers of Muc2/IL-10 ${ }^{\mathrm{DKO}}$ mice were in line with Mendelian predictions, indicating no prenatal selection or death before 4 weeks. However, high mortality was observed at 4 weeks of age (Figure 1a). Seven of the 23 mice died between 3 and 4 weeks of age, and three others between 4 and 5 weeks (Figure 1a). Therefore, we killed two groups of five and seven Muc2/IL-10 ${ }^{\mathrm{DKO}}$ mice at 4 and 5 weeks, respectively. These mice did not contribute to the survival curve. The only surviving mouse was euthanized at 7 weeks of age and was excluded from analysis. None of the WT, IL- $10^{-1-}$ and Muc2 $^{-1-}$ mice died during the time frame studied. These animals most probably did not die of sepsis caused by translocation of bacteria as assessed by blood culture and analysis of splenic tissue ${ }^{27}$ (data not shown).

A significant difference $(P=0.008)$ in body weight was detected at 4 weeks between WT and Muc2/IL- $10^{\mathrm{DKO}}$ mice. Interestingly, WT mice significantly gained weight between 4 and 5 weeks of age $(17.2 \pm 1.1$ vs $22.3 \pm 1.4 \mathrm{~g}$, respectively; $P=0.016)$, whereas weight of Muc2/IL- $10^{\mathrm{DKO}}$ mice stabilized (6.4 \pm 0.4 vs $6.7 \pm 0.3 \mathrm{~g}$, respectively; $P=0.53)$. Similarly, although less severe than Muc2/IL-10 ${ }^{\text {DKO }}$ mice, 5-week-old Muc2 ${ }^{-1-}$ mice showed significant growth retardation (Figure 1b). Only at 8 weeks, WT mice were significantly heavier than IL$10^{-1-}$ mice, $\quad(25.6 \pm 0.2$ vs $24.2 \pm 0.34 \mathrm{~g}$, respectively; $P=0.005)$.

Furthermore, at 4 weeks, all Muc2/IL- $10^{\mathrm{DKO}}$ mice showed diarrhea and gross bleeding, which persisted until 5 weeks of age, unlike the control WT littermates. DAI scores for Muc2/
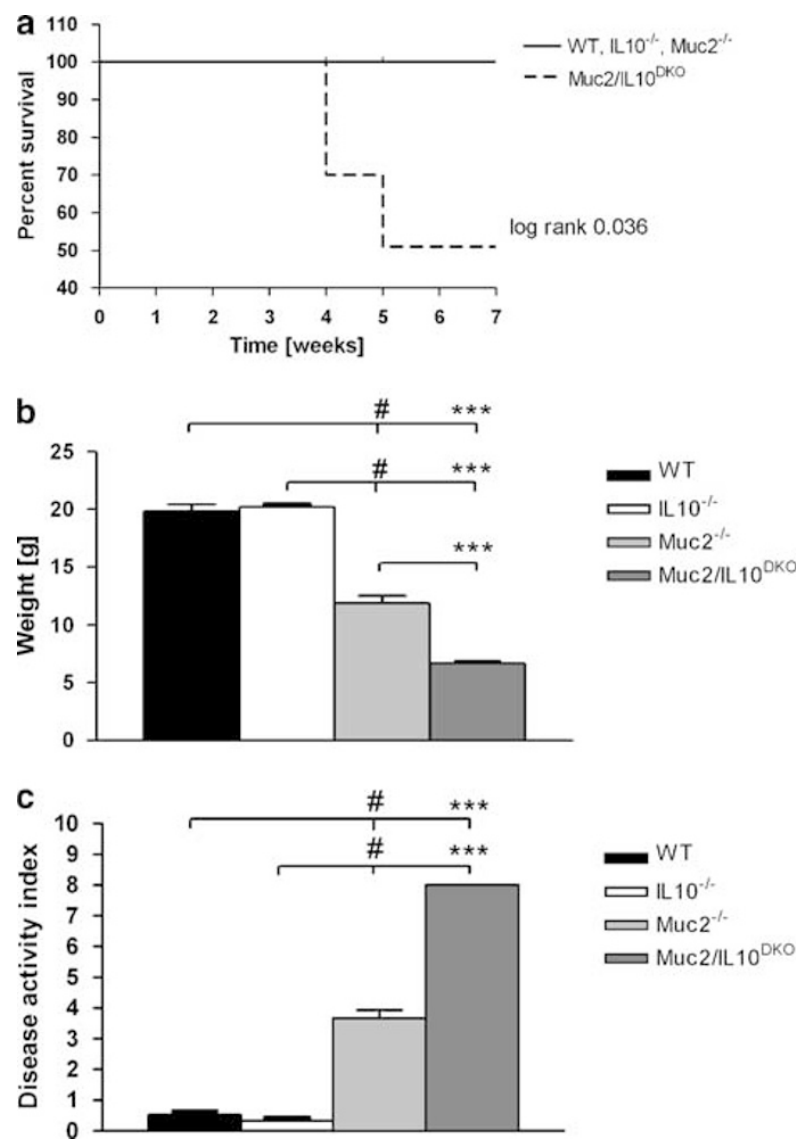

Figure 1 Appearance and clinical symptoms of WT, IL-10 $0^{-1-}$, Muc2 $2^{-1-}$ and Muc2/IL-10 $0^{\mathrm{DKO}}$ mice. (a) Survival of the Muc2/IL-10 ${ }^{\mathrm{DKO}}$ mice. None of the WT, IL- $10^{-1-}$ and Muc2 ${ }^{-1-}$ mice died during the time frame studied. (b) Body weight of the different mice at 5 weeks. $\left({ }^{*} P<0.0001 ;{ }^{\#} P=0.0002\right)$ (c) Disease activity index (DAI) is an accumulative score as adapted from Cooper et $a l^{20}\left({ }^{\star} P<0.0001 ;{ }^{\#} P=0.0002\right)$. Error bars indicate the s.e.m.

IL-10 ${ }^{\mathrm{DKO}}$ mice were therefore significantly higher (Figure 1c). Differences in DAI scores between WT and IL- $10^{-1-}$ mice are due to variances in weight differences within a group, and are nonsignificant.

\section{Morphological Analysis}

In line with the clinical observations, there were no morphological differences in the proximal or distal colon between WT and IL- $10^{-1-}$ mice at 5 up to 16 weeks. At 34 weeks, the distal colon of IL-10 $0^{-1-}$ mice showed mild inflammation characterized by mucosal thickening, influx of inflammatory cells and overall mild distortion of the architecture (data not shown). The colonic morphology of Muc2/IL-10 ${ }^{\mathrm{DKO}}$ animals differed considerably from their WT littermates (Figure 2a and $\mathrm{b}$, proximal colon and Figure $2 \mathrm{e}$ and $\mathrm{f}$, distal colon), and both IL- $10^{-1-}$ and Muc2 $2^{-1-}$ mice. Apart from absence of the characteristic bell-shaped goblet cells, the Muc2/IL-10 ${ }^{\text {DKO }}$ mice showed crypt hyperplasia, infiltration, flattening of epithelial cells and distortion of the lamina propria at 4 weeks in the proximal colon. Furthermore, apoptotic bodies were 

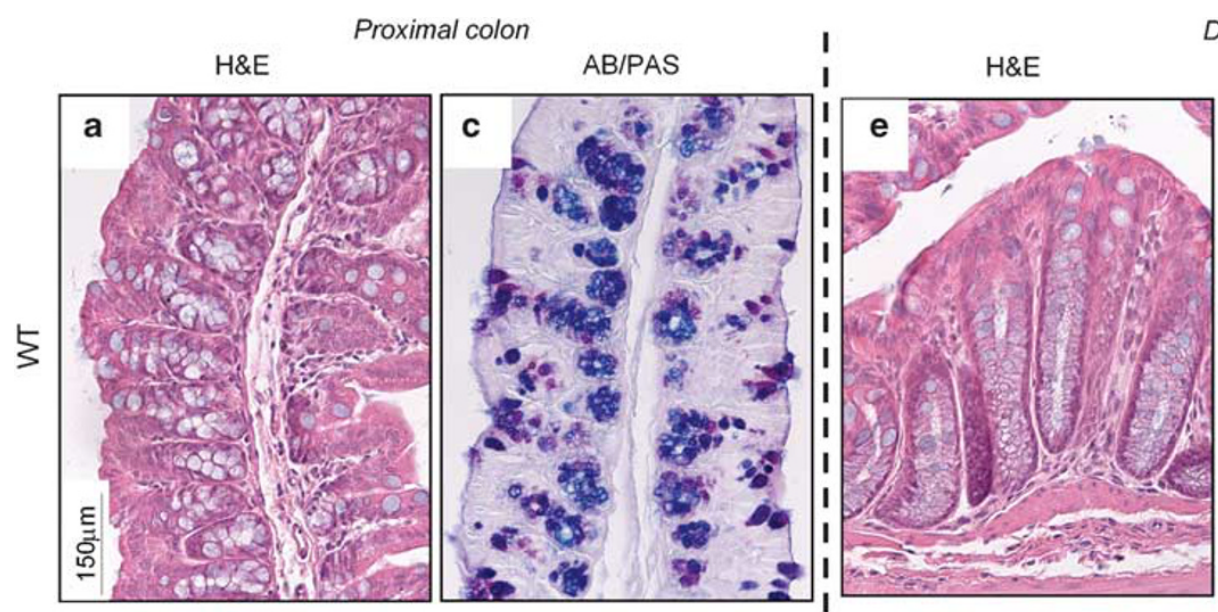

Distal colon
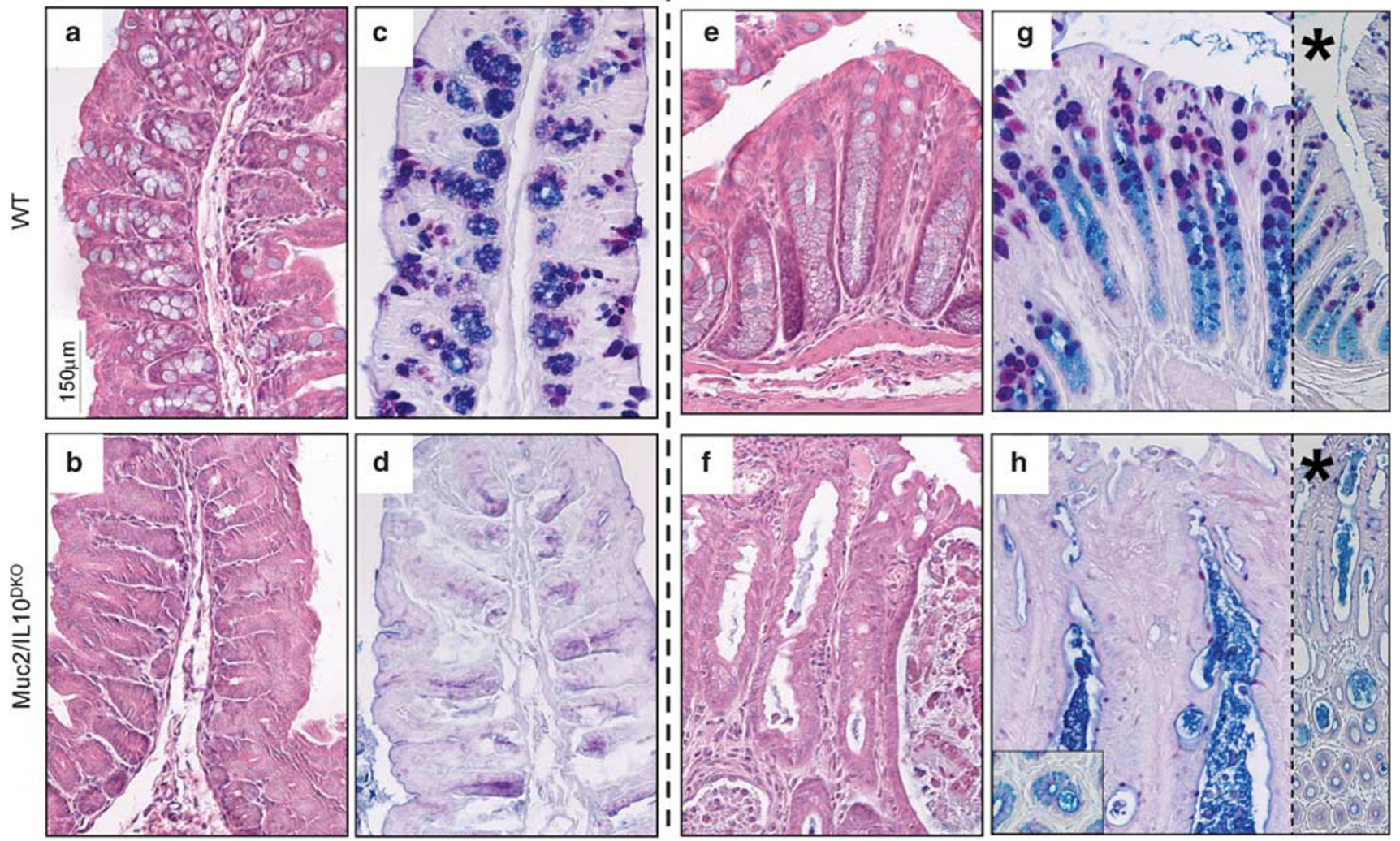

Figure 2 Colonic morphology of the WT and Muc2/IL-10 DKO mice. Representative sections of the colon are depicted at 5 weeks (a-d, proximal colon; e-h, distal colon). (a and $\mathbf{b}$, and $\mathbf{e}$ and $\mathbf{f}$ ) Represent hematoxylin and eosin stainings whereas (c and $\mathbf{d}$ ) and ( $\mathbf{g}$ and $\mathbf{h}$ ) represent AB-PAS stainings. (a and $\mathbf{c}$, and $\mathbf{e}$ and $\mathbf{g}$ ) Represent proximal colon and distal colon, respectively of the WT mice. (b and $\mathbf{d}$, and $\mathbf{f}$ and $\mathbf{h}$ ) Represent proximal colon and distal colon, respectively of the Muc2/IL-10 ${ }^{\mathrm{DKO}}$ mice. The inset in (h) shows a close-up of goblet cells in the crypts of Muc2/IL-10 ${ }^{\mathrm{DKO}}$ mice. The panels on the right-hand side with an asterisk represent a lower magnification of the distal colon.

frequently observed along the crypts in the Muc2/IL-10 ${ }^{\mathrm{DKO}}$, which were hardly seen in all the other types of mice (Figure 3). The overall colonic damage increased along the proximal-distal axis and in addition to the histopathology described above, severe ulceration and crypt abscesses were observed in the distal colon of Muc2/IL- $10^{\mathrm{DKO}}$ mice. These characteristics were most pronounced in older mice (5 weeks) and more severe than in the Muc2 $2^{-1-}$ mice.

The presence of goblet cells in the colonic mucosa was determined by AB/PAS staining, which stains glycoproteins. Goblet cells of the proximal and distal colon were mainly positive for $\mathrm{AB}$ at the crypt bases whereas PAS-positive cells were concentrated higher in the crypts in WT and IL-10 ${ }^{-1-}$ mice (Figure $2 \mathrm{c}$ and g, WT proximal and distal colon, respectively, 5 weeks). The proximal colon of Muc $2 / \mathrm{IL}-10^{\mathrm{DKO}}$ mice hardly contained any PAS-positive cells and no ABpositive cells (Figure 2d, 5 weeks). The distal colon of the Muc2/IL-10 DKO mice, however, contained few small, condensed, PAS-positive cells along the crypts and AB-positive cells at the base of the crypts (Figure $2 \mathrm{~h}, 5$ weeks).

Epithelial proliferation was studied by immunohistochemical staining of incorporated BrdU. WT and

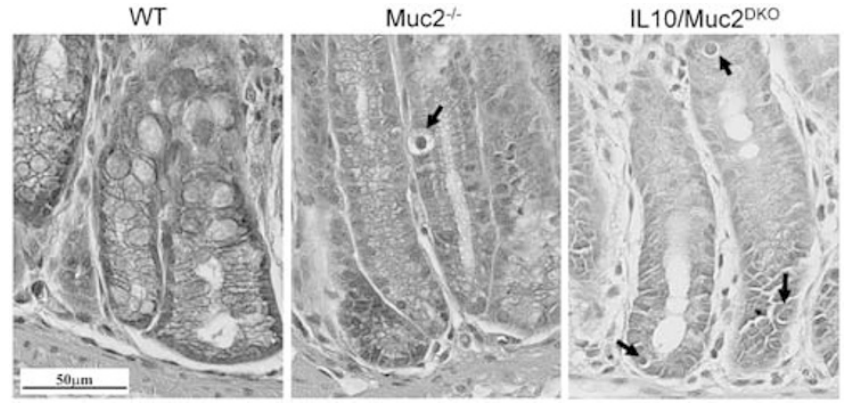

Figure 3 Increase of apoptosis in the crypts of the Muc2/IL-10 DKo. The different panels represent sections of the distal colon of the different types of mice, 5 weeks of age. Arrows indicate presence of apoptotic bodies.

IL-10 $0^{-1-}$ mice did not differ in distribution or localization of BrdU-positive cells in the proximal and distal colon at all ages (Supplementary Figure 1D and E showing distal colon of WT and IL-10 $10^{-1-}, 5$ weeks of age). In WT mice, BrdU-positive cells were localized at the bases, and up to $1 / 3$ of the crypts in the proximal and distal colon (Figure $4 \mathrm{a}-\mathrm{c}$, respectively, 5 weeks old). In both the proximal and distal colon of all Muc2/IL- $10^{\mathrm{DKO}}$ mice, however, the number of BrdU-positive 

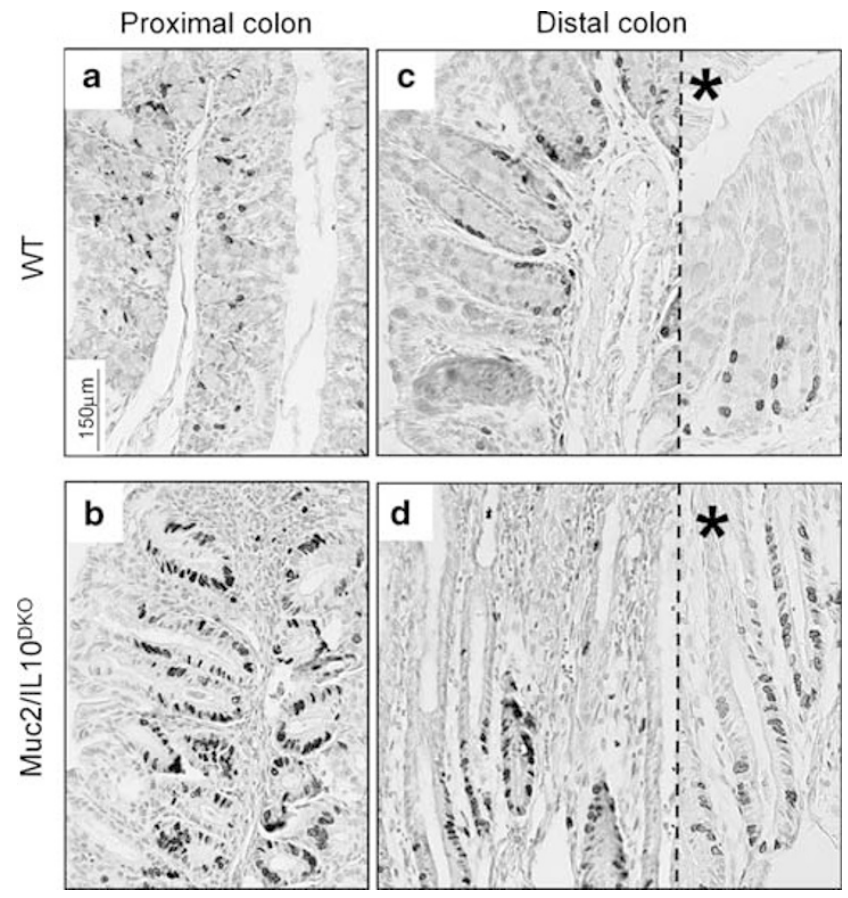

Figure 4 Increased proliferation in the colonic crypts of Muc2/IL-10 DKO mice. BrdU staining of the proximal ( $\mathbf{a}$ and $\mathbf{b}$ ) and the distal ( $\mathbf{c}$ and $\mathbf{d}$ ) colon. In WT mice, BrdU-positive cells were localized at the base of the crypts in both proximal and distal colon (a and $\mathbf{c}$, respectively). The Muc2/IL-10 mice, however, showed an increase in the number of BrdU-positive cells in both the proximal and the distal colon and these cells extended halfway up the crypt (b and $\mathbf{d}$, respectively). The panels on the right-hand side with an asterisk represent a higher magnification of the crypts in the distal colon.

cells was higher as early as 4 weeks and these cells extended up into crypts (Figure 4b-d, respectively, 5 weeks of age).

\section{Expression of Goblet-Cell Markers}

The intestinal epithelium of Muc2/IL-10 ${ }^{\mathrm{DKO}}$ mice showed altered $\mathrm{AB}$ and PAS staining, as described above. Therefore, we ascertained the presence and maturation of the goblet-cell lineage in the different mouse models by determining the expression of Tff3 and Muc4. ${ }^{24,25,28,29}$ Muc4 and Tff3 were expressed by goblet cells, concentrated mainly in the upper portion of the crypts, in both the proximal and distal colon of WT mice (Figure 5a and c, Tff3, blue panel; Figure 5e and g, Muc4, orange panel). The staining pattern of Muc4 in the IL$10^{-1-}$ mice corresponded to the staining pattern of this marker in the WT mice (Figure 5k and 1). Interestingly, there was a decrease of Muc4-positive goblet cells in both the proximal and distal colon of Muc2 $2^{-l-}$ mice (Figure $5 \mathrm{i}$ and $\mathrm{j}$ ). Goblet cells were virtually absent in the proximal colon of the Muc2/IL- $10^{\text {DKO }}$ mice using both the goblet-cell markers Tff3 and Muc4 (Figure 5b and f, respectively). Interestingly, both Tff3 and Muc4 were detectable in goblet cells in the distal colon of the Muc2/IL- $10^{\mathrm{DKO}}$ mice (Figure $5 \mathrm{~d}$ and h, respectively), but in smaller numbers compared to WT mice. Furthermore, the goblet cells in the distal colon of the Muc2/ IL- $10^{\text {DKO }}$ mice, as observed in the Muc2 ${ }^{-/-}$mice, had lost their characteristic goblet-like shape (inset of Figure 5c, WT mice $v s$ inset of Figure 5d, Muc2/IL-10 ${ }^{\mathrm{DKO}}$ mice).

\section{Expression of the Muc1, Muc3 and Muc4 Genes}

The expression of the mucins, Muc1, Muc3 and Muc4, was quantified at RNA level by real-time RT-PCR in the distal colon of each type of mice (Figure 6). Mucl expression in each type of knockout mouse did not differ compared to control WT littermates. In contrast, Muc3 mRNA was decreased in the distal colon of Muc2 ${ }^{-1-}$ mice and significantly decreased in the Muc2/IL-10 ${ }^{\text {DKO }}$ mice compared to WT littermates. Corresponding to the Muc4 protein expression pattern found in the different types of mice (Figure 5, orange panel), there were no significant changes in Muc4 mRNA levels between the IL- $10^{-l-}$, Muc2 ${ }^{-1-}$ and WT mice, whereas the mRNA expression was significantly decreased in the Muc2/IL- $10^{\mathrm{DKO}}$ mice.

\section{Increased Lymphocyte Infiltration and Expression of Inflammatory Cytokines}

Lymphocyte infiltration into the colon was evaluated using an anti-CD3 antibody. In both the proximal and the distal colon of WT and IL-10 $0^{-1-}$ mice, single cells in the lamina propria were detected (Figure 7a). In the $\mathrm{Muc}^{-1-}$ mice, there was an increase of lymphocyte infiltration, that is CD3positive cells, in parts of the colon where there were loss of epithelial cells ${ }^{14}$ (Figure 7a). The Muc2/IL-10 $0^{\text {DKO }}$ mice showed increased lymphocyte infiltration (compared to both WT and $\mathrm{Muc}^{-1-}$ mice), characterized by large clusters in both the proximal and the distal colon compared to WT and Muc2 $^{-1-}$ mice (Figure 7a, Muc2/IL-10 ${ }^{\text {DKO }}$ mice, showing distal colon, 5 weeks). In addition, there was an increase of lymphocytes along the proximal-distal axis, coinciding with the increase in histological damage seen in the colon of the Muc2/IL-10 ${ }^{\mathrm{DKO}}$ mice. Furthermore, mRNA expression showed a significant increase in the inflammatory cytokines TNF- $\alpha$, IL- $1 \beta$ and IL- 6 in the distal colon of Muc2/IL- $10^{\text {DKO }}$ mice compared to WT mice (Figure 7b, 5 weeks). The mRNA expression levels of these cytokines were not significantly upregulated in $\mathrm{IL}-10^{-1-}$ and Muc2 $2^{-1-}$ mice compared to their WT littermates, 5 weeks of age (Figure $7 \mathrm{~b}$ ). In accordance with the real-time PCR data, expression of inflammatory cytokines IL-12p70, TNF- $\alpha$, IFN- $\gamma$, MIP- 1 and IL-6 in the serum of Muc2/IL-10 ${ }^{\text {DKO }}$ mice was increased compared to WT mice as early as 4 weeks, which persisted as the mice aged (Figure 7c, 5 weeks).

\section{DISCUSSION}

In this paper, we showed that both immunological and epithelial deficiencies in mice lacking the anti-inflammatory cytokine IL-10 and mucosal protector mucin 2 sensitize the intestine to severe colonic inflammation. The morphological, phenotypic and functional alterations observed make this murine model pertinent to study multiple aspects of IBD. 

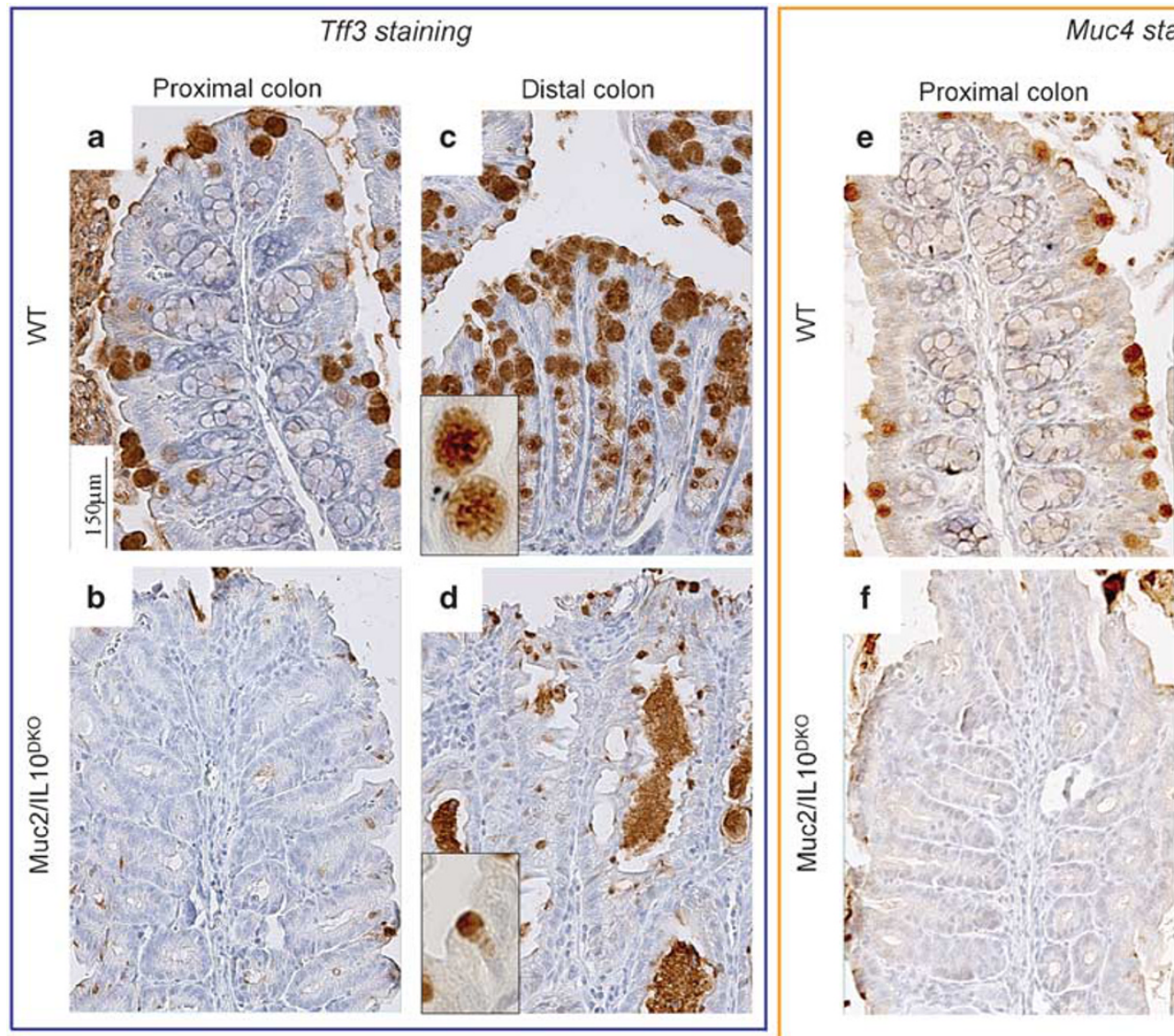

\section{Muc4 staining}
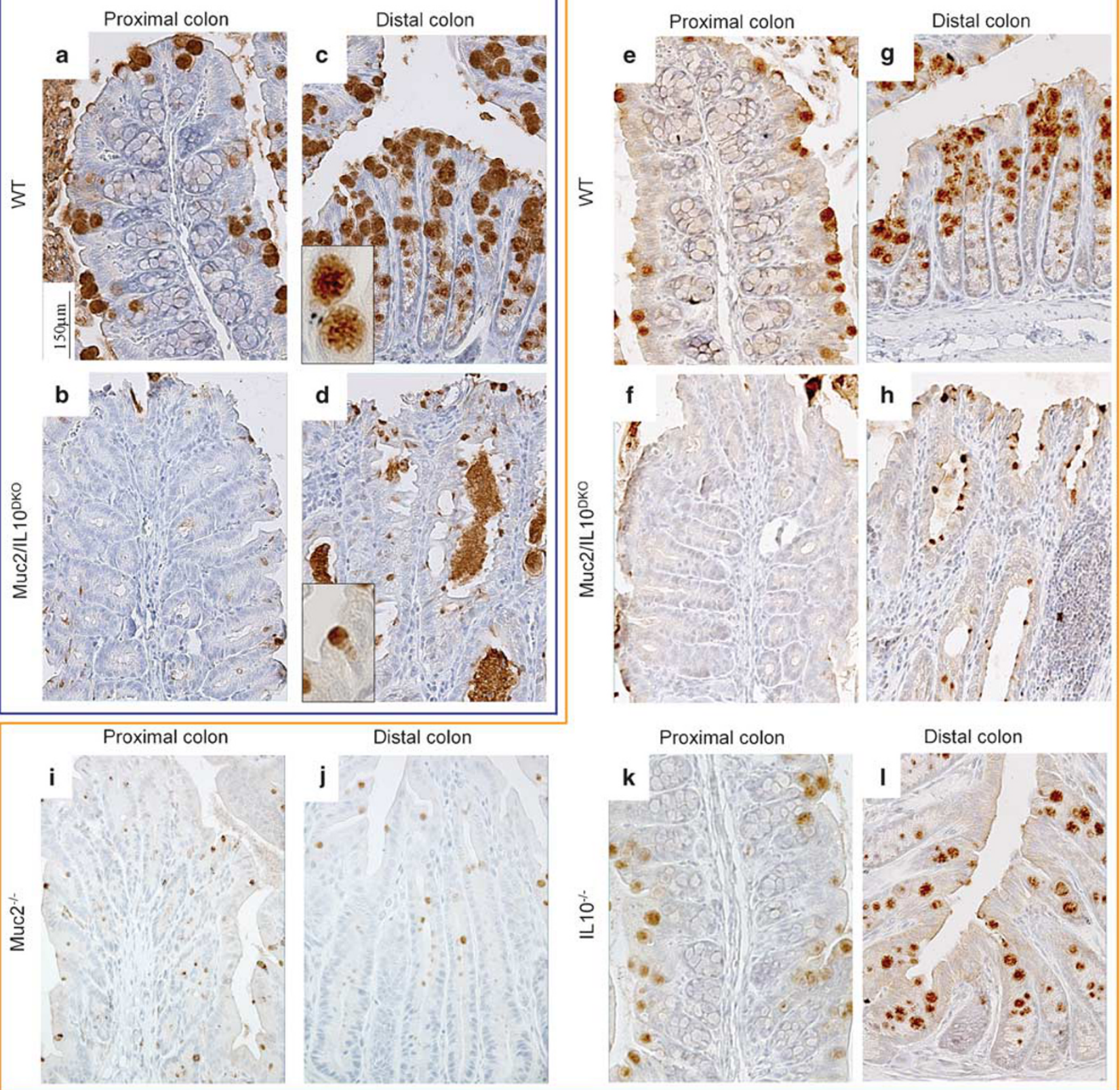

Figure 5 Localization of Tff3 and Muc4 protein expression in the colon. Tff3 (blue panel) was expressed by goblet cells in the proximal colon (a) of WT mice, but was virtually absent in the proximal colon (b) of Muc2/IL-10 ${ }^{\mathrm{DKO}}$ mice. In the distal colon, Tff 3 was present in the goblet cells (c) of WT mice and (d) Muc2/ IL-10 ${ }^{\mathrm{DKO}}$ mice. (e) Muc4 (orange panel) was expressed by the proximal colon of WT mice, but is hardly detectable in the proximal colon (f) of Muc2/IL-10 ${ }^{\mathrm{DKO}}$ mice. Muc4 was expressed by goblet cells in the (g) distal colon of both WT and (h) Muc2/IL-10 ${ }^{\text {DKO }}$ mice. In the Muc2 ${ }^{-1-}$ mice, Muc4 was expressed by goblet cells in both the proximal (i) and the distal colon (j), but in fewer numbers compared to WT mice. The staining for Muc4 in the IL-10 ${ }^{-1-}$ mice corresponded to the staining pattern in the WT mice proximal colon (k) and distal colon (I).

One of the most important phenotypic change observed in Muc2/IL- $10^{\text {DKO }}$ mice was growth retardation, which persisted with age and which was more severe than that in $\mathrm{Muc}^{-1-}$ mice. It is known that the most prominent factor affecting growth is inadequate nutritional intake. ${ }^{30}$ Moreover, growth retardation in IBD patients can be correlated with an increase in systemic TNF- $\alpha$ and IL-6 levels. ${ }^{31,32}$ The body- weight alterations observed both in $\mathrm{Muc2}^{-1-}$ and Muc2/ IL- $10^{\text {DKO }}$ mice support this paradigm, as the weights of these mice correlated with the severity of inflammation. In addition, Muc2/IL-10 ${ }^{\mathrm{DKO}}$ animals also show occult blood loss to gross bleeding and diarrhea at 4 weeks, unlike the WT and $\mathrm{IL}-10^{-1-}$ mice. In this respect, the Muc2/IL-10 ${ }^{\mathrm{DKO}}$ mouse model seems to recapitulate symptoms well established in 


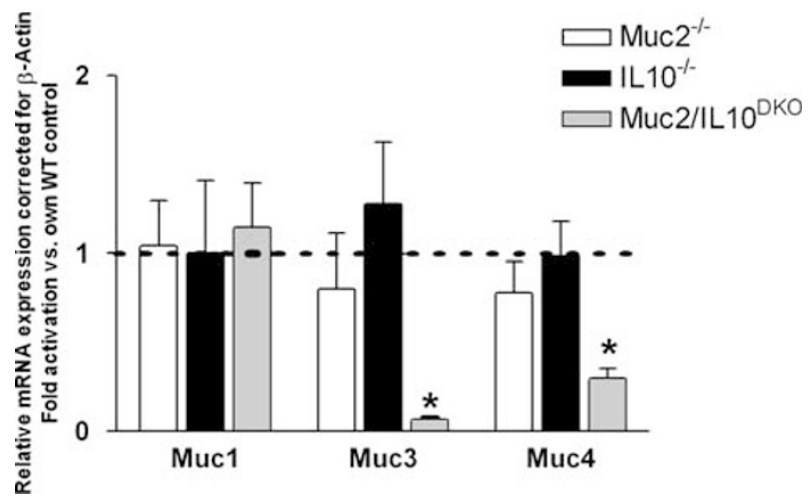

Figure 6 Expression of Muc1, Muc3 and Muc4 mRNA in the distal colon. The mRNA levels of Muc1, Muc3 and Muc4 in Muc2 ${ }^{-1-}, \mathrm{IL}-10^{-/-}$and DKO mice were quantified using real-time RT-PCR. Expressed values ( \pm s.e.m.) are given relative to control values (wild-type littermates), which were arbitrarily set on $1\left({ }^{*} P<0.02\right)$. children with IBD, for example, growth failure, diarrhea, rectal bleeding and weight loss. ${ }^{33}$ However, this mouse model is severe, as the Muc2/IL-10 ${ }^{\text {KKO }}$ mice showed a high mortality rate, in contrast to the Muc2 $2^{-1-}, \mathrm{IL}_{-} 10^{-1-}$ and WT littermates, of which none died during the current time frame (ie up to 7 weeks of age). Histological analysis of the colon of the Muc2/IL-10 ${ }^{\mathrm{DKO}}$ mice additionally confirmed severe inflammation. The Muc2/IL-10 ${ }^{\mathrm{DKO}}$ mice showed distinct crypt hyperplasia, inflammatory cell infiltration, flattening of epithelial cells and distortion of the lamina propria, which aggravated as the mice aged. This pathology was even more severe in the distal colon, which in addition to the above characteristics, also showed crypt abscesses.

All together, these clinical and histological symptoms indicate that the Muc2/IL-10 ${ }^{\mathrm{DKO}}$ mice constitute a good model to study several aspects of IBD, as described above, which a

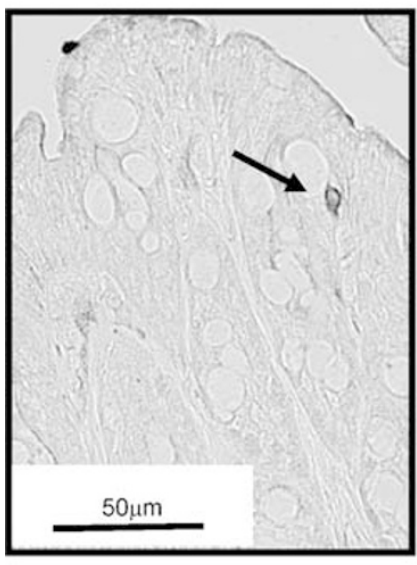

b

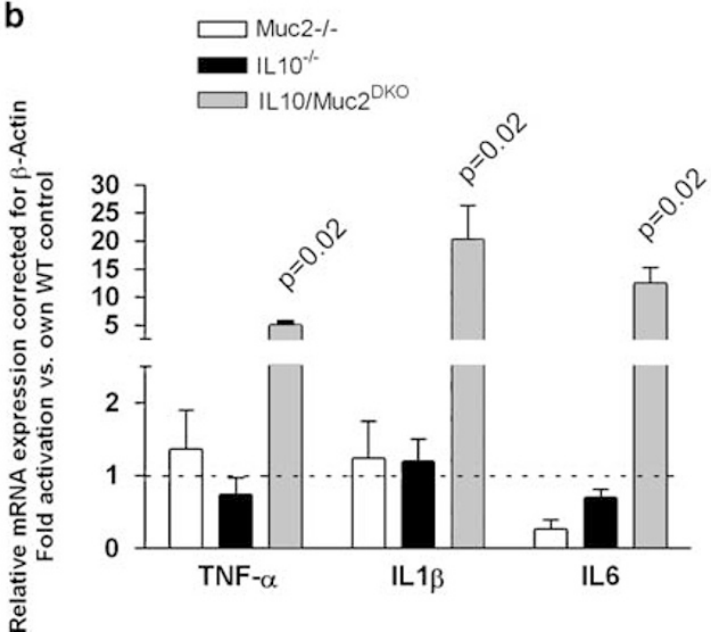

Muc2 $\%$
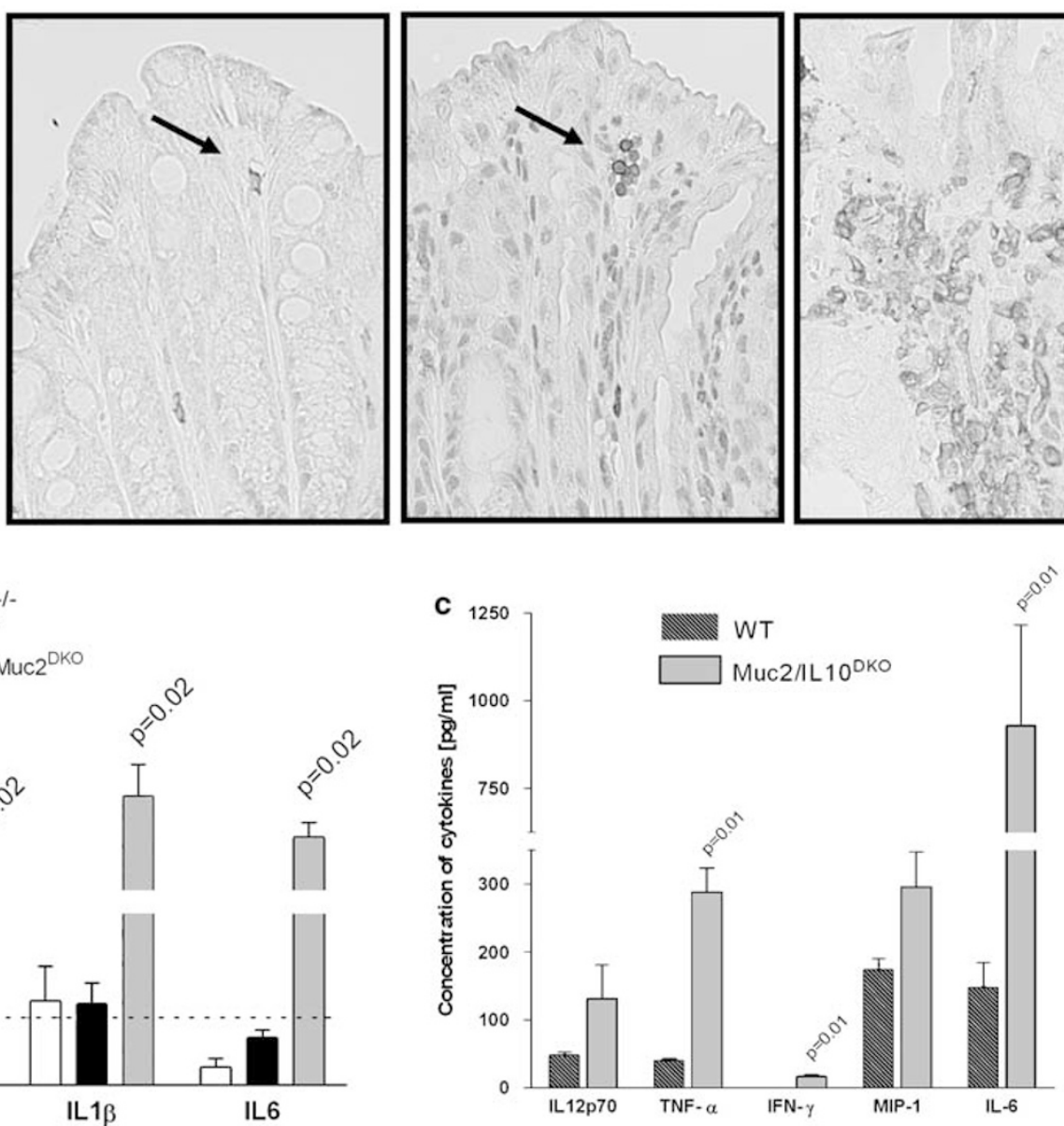

Figure 7 Increased lymphocyte infiltration and expression of inflammatory cytokines in Muc2/IL-10 ${ }^{\mathrm{DKO}}$ mice. (a) Representative sections presenting lymphocyte infiltration into the distal colon of WT, IL-10 $0^{-1-}$, Muc2 $^{-1-}$ and Muc2/IL-10 ${ }^{\mathrm{DKO}}$ mice detected using an anti-CD3 antibody. Arrows in the first three panels indicate presence of $\mathrm{CD}^{+}$cells. (b) Real-time PCR data showed a significant upregulation of the inflammatory cytokines TNF- $\alpha$, IL-1 $\beta$ and IL- 6 $(P=0.02)$ in the distal colon of Muc2/IL-10 ${ }^{\mathrm{DKO}}$ mice (dotted bars) compared to WT mice whereas no significant changes were detected in both IL-10 ${ }^{-/-}$ (black bars) and Muc2 ${ }^{-1-}$ (white bars) mice, compared to WT mice, 5 weeks of age. (c) Analysis of the serum showed an increase in expression of inflammatory cytokines IL-12p70, TNF- $\alpha$, IFN- $\gamma$, MIP-1 and IL- 6 in the serum of Muc2/IL-10 ${ }^{\text {DKO }}$ mice (dotted bars) compared to WT mice (striped bars). Muc2/IL-10 ${ }^{\mathrm{DKO}}, n=7 ; \mathrm{WT}, n=4$; error bars indicate the s.e.m. 
has an earlier onset and is more severe compared to both IL- $10^{-1-}$ and $\mathrm{Muc}^{-1-}$ mice.

We investigated the presence and maturation of goblet cells in the mucosa in the Muc2/IL-10 ${ }^{\mathrm{DKO}}$ through (i) AB/PAS (ii) Tff3 and (iii) Muc4 staining. Tff3-positive goblet cells were present throughout the entire large intestine in WT, IL-10 ${ }^{-/-}$ and $\mathrm{Muc}^{-1-}$ mice. Contrastingly, in the Muc2/IL-10 ${ }^{\text {DKO }}$ mice, Tff3-positive goblet cells were present only in the distal colon and were absent in the proximal colon. This pattern coincided with the pattern for the PAS-positive staining of cells in the intestine. Muc4 was present in fully differentiated goblet cells (higher in the crypts) throughout the colon of the WT mice. This pattern is in agreement with our previous data recently published in WT mice. ${ }^{25}$ Interestingly, diminished differentiation of goblet cells in the colon of $\mathrm{Muc}^{-1-}$ and Muc2/IL-10 ${ }^{\text {DKO }}$ mice was confirmed by a decrease in Muc4 expression. There was even an almost complete loss of Muc4 protein expression in the goblet cells of the proximal colon in both these types of knockout mice. Loss of Muc4 expression in the colon combined with the significant increase in proliferation in the colon in both the Muc2 ${ }^{-/-}$and the Muc2/ IL- $10^{\mathrm{DKO}}$ mice is in line with a previous study, in which rat Muc4 was associated with highly/terminally differentiated, non-proliferating epithelial cells. ${ }^{34}$ We propose that loss of Muc4 expression is not correlated with the amount of damage (proximal $v s$ distal colon), but to a difference in gobletcell differentiation stage. More specifically, in the proximal colon, both Tff3 and Muc4 are expressed only by the terminally differentiated goblet cells in the upper part of the crypt. In the distal colon, the expression of both these markers is concentrated in the upper part of the crypts but goblet cells throughout the crypts also stain positive for these markers. Changes in the proliferation-differentiation balance might therefore affect the expression of these markers differently in both regions. This mechanism needs to be further investigated, but already transcription factors, which control intestinal differentiation, may be of interest, that is, Cdx-1 and $\mathrm{Cdx}-2$ were recently identified as important regulators of Muc4 expression in mice colon. ${ }^{25}$ In addition, loss of differentiation of intestinal cells during damage, in general, has been shown in several animal models, by downregulation of distinct enterocyte-specific proteins. ${ }^{14,35,36}$ The ABpositive cells at the crypt bases in the distal colon in the Muc2/IL-10 ${ }^{\text {DKO }}$ mice could indicate transient expression of other mucins known to be expressed in the intestine or de novo expression of Muc6 mRNA as described for the Muc2 $^{-/-}$mice. $^{14}$

Recent studies have implicated a role for membranebound mucins in signaling, contributing to the modulation of intestinal-cell proliferation and differentiation as reviewed by ${ }^{37}$ Hollingsworth and Swanson. Both proliferation and differentiation are affected in the $\mathrm{Muc2}^{-1-}$ and Muc2/ IL- $10^{\mathrm{DKO}}$ mice, as determined by the increased proliferation of the distal colon as well as loss of differentiation in the goblet-cell lineage. Therefore, we investigated the expression of the two well-characterized membrane mucins, Mucl and Muc3, in these mice. Mucl mRNA was unaffected in all types of knockout mice, compared to WT mice. In contrast, Muc3 mRNA, although modestly downregulated in $\mathrm{Muc2}^{-1-}$ mice, was significantly downregulated in Muc2/IL-10 ${ }^{\text {DKO }}$ mice. Muc3, specifically, has shown to stimulate cell migration, inhibit apoptosis and accelerated wound healing. ${ }^{38}$ Significant downregulation of this mucin in the Muc2/IL- $10^{\mathrm{DKO}}$ could therefore partly explain and/or contribute to the severity in histological damage observed in these mice, compared to the singleknockout mice.

An increase in exposure to luminal antigens due to a diminished mucus layer (decrease of Muc2) could initiate inflammation. The Muc2/IL-10 ${ }^{\mathrm{DKO}}$ mice showed increased lymphocyte infiltration, that is CD3-positive cells, throughout the colon, which was more severe compared to $\mathrm{Muc2}^{-1-}$ mice. The increased infiltration coincided with upregulation of mRNA for the inflammatory cytokines TNF- $\alpha$, IL- $1 \beta$ and IL- 6 in the distal colon of Muc2/IL-10 ${ }^{\text {DKO }}$ at 5 weeks of age. In addition, TNF- $\alpha$, IFN- $\gamma$ and IL- 6 were also significantly upregulated systemically in the Muc2/IL- $10^{\mathrm{DKO}}$ at 5 weeks of age. These pro-inflammatory cytokines play a pivotal role in the pathogenesis of IBD. ${ }^{1,3,4}$ Furthermore, previous studies have shown that TNF- $\alpha / \mathrm{IL}-1 \beta / \mathrm{IL}-6$ are upregulated in both the IL- $10^{-1-}$ and the Muc2 $2^{-1-}$ mice, suggesting that similar types of immune responses are responsible for the inflammation observed. ${ }^{14,39}$ Previous studies also demonstrated that IL-10 is essential for producing transforming growth factor beta (TGF- $\beta$ ) and that, in agreement, IL-10 ${ }^{-1-}$ mice lack TGF- $\beta$ signaling. ${ }^{40,41}$ Lack of both IL-10 and TGF- $\beta$ signaling in the Muc2/IL- $10^{\text {DKO }}$ mice could explain the excessive inflammation in these mice compared to Muc2 $^{-1-}$ mice. In addition, the enhanced histological damage could be attributed to upregulation of IL-6, which has been shown to degrade the epithelial barrier and increase cell proliferation. ${ }^{42}$

To summarize, the Muc2/IL-10 ${ }^{\mathrm{DKO}}$ mouse clearly demonstrates that combined abnormalities in immunoregulatory and epithelial factors greatly accelerate and exacerbate the phenotype of colonic inflammation. In fact, in our facility, the IL-10 $0^{-1-}$ mice show no distinct clinical or histological signs of colitis at 5 weeks of age indicating that the mucus barrier offers enough protection under unchallenged conditions. Muc2 $2^{-1-}$ mice, however, show abnormal morphology marked by thickening of the gut mucosa, flattening and ulceration of epithelial cells, general loss of architecture, increase of inflammatory cells, increase in proliferation and decrease of cell differentiation in the colon as described previously. ${ }^{14}$ Therefore, this study provides evidence that in case of Muc2 deficiency, the anti-inflammatory cytokine IL-10 can control epithelial damage, though to a limited extent. Most importantly, these data show that the intestinal mucus layer in the intestine, more specifically its constituent mucin Muc2, is indispensable in mucosal protection, 
suggesting that impaired mucus barrier function is a major factor for the development of colitis.

Supplementary Information accompanies the paper on the Laboratory Investigation website (http://www.laboratoryinvestigation.org)

\section{ACKNOWLEDGEMENTS}

Sophia Foundation for Scientific Research, Rotterdam, The Netherlands Organization for Scientific Research, The Hague, The Foundation 'De Drie Lichten', all situated in the Netherlands, The Association François Aupetit, Paris, France and the National Institutes of Health, United States are gratefully acknowledged for their financial support. We would like to thank W Chung, IMA Louwers and LF de Ruiter for their excellent technical assistance.

1. Bouma G, Strober W. The immunological and genetic basis of inflammatory bowel disease. Nat Rev Immunol 2003;3:521-533.

2. Einerhand AW, Renes IB, Makkink MK, et al. Role of mucins in inflammatory bowel disease: important lessons from experimental models. Eur J Gastroenterol Hepatol 2002;14:757-765.

3. Fiocchi C. Inflammatory bowel disease: etiology and pathogenesis. Gastroenterology 1998;115:182-205.

4. Strober W, Fuss IJ, Blumberg RS. The immunology of mucosal models of inflammation. Annu Rev Immunol 2002;20:495-549.

5. Elson CO, Sartor RB, Tennyson GS, et al. Experimental models of inflammatory bowel disease. Gastroenterology 1995;109:1344-1367.

6. Kuhn R, Lohler J, Rennick D, et al. Interleukin-10-deficient mice develop chronic enterocolitis. Cell 1993;75:263-274.

7. Mahler M, Leiter EH. Genetic and environmental context determines the course of colitis developing in IL-10-deficient mice. Inflamm Bowel Dis 2002;8:347-355.

8. Sellon RK, Tonkonogy S, Schultz M, et al. Resident enteric bacteria are necessary for development of spontaneous colitis and immune system activation in interleukin-10-deficient mice. Infect Immun 1998;66:5224-5231.

9. Herrmann A, Lindell $\mathrm{G}$, Nordman $\mathrm{H}$, et al. The insoluble glycoprotein complex from human colon contains two MUC2 subunits of different size. Biochem Soc Trans 1995;23:535S

10. van Klinken BJ, Einerhand AW, Duits LA, et al. Gastrointestinal expression and partial CDNA cloning of murine Muc2. Am J Physiol 1999;276:115-124.

11. Makkink MK, Schwerbrock NM, Mahler $M$, et al. Fate of goblet cells in experimental colitis. Dig Dis Sci 2002;47:2286-2297.

12. Tytgat KM, Buller HA, Opdam FJ, et al. Biosynthesis of human colonic mucin: Muc2 is the prominent secretory mucin. Gastroenterology 1994;107:1352-1363.

13. Tytgat KM, Bovelander FJ, Opdam FJ, et al. Biosynthesis of rat MUC2 in colon and its analogy with human MUC2. Biochem J 1995;309(Part 1): 221-229.

14. van der Sluis M, de Koning BAE, de Bruijn ACJM, et al. Muc2-deficient mice spontaneously develop colitis, indicating that MUC2 is critical for colonic protection. Gastroenterology 2006;131:117-129.

15. Tytgat KM, van der Wal JW, Einerhand AW, et al. Muc2 in ulcerative colitis: a quantitative study. Biochem Soc Trans 1995;23:531S.

16. Van Klinken BJ, Van der Wal JW, Einerhand AW, et al. Sulphation and secretion of the predominant secretory human colonic mucin MUC2 in ulcerative colitis. Gut 1999:44:387-393.

17. Berg DJ, Davidson N, Kuhn R, et al. Enterocolitis and colon cancer in interleukin-10-deficient mice are associated with aberrant cytokine production and CD4(+) TH1-like responses. J Clin Invest 1996;98:10101020.

18. Velcich A, Yang W, Heyer J, et al. Colorectal cancer in mice genetically deficient in the mucin Muc2. Science 2002;295:1726-1729.

19. Spencer DM, Veldman GM, Banerjee $S$, et al. Distinct inflammatory mechanisms mediate early vs late colitis in mice. Gastroenterology 2002;122:94-105.

20. Cooper HS, Murthy SN, Shah RS, et al. Clinicopathologic study of dextran sulfate sodium experimental murine colitis. Lab Invest 1993;69:238-249.
21. Wahba N. An ortho-tolidine hydrochloride test for the detection of occult blood in faeces without dietary restrictions. J Clin Pathol 1965;18:687-688.

22. Renes IB, Verburg M, Bulsing NP, et al. Protection of the Peyer's patchassociated crypt and villus epithelium against methotrexate-induced damage is based on its distinct regulation of proliferation. J Pathol 2002;198:60-68.

23. Boshuizen JA, Reimerink JH, Korteland-van Male AM, et al. Changes in small intestinal homeostasis, morphology, and gene expression during rotavirus infection of infant mice. J Virol 2003;77:13005-13016.

24. Mashimo H, Wu DC, Podolsky DK, et al. Impaired defense of intestinal mucosa in mice lacking intestinal trefoil factor. Science 1996;274: 262-265.

25. Jonckheere N, Vincent A, Perrais M, et al. The human mucin MUC4 is transcriptionally regulated by caudal-related homeobox, hepatocyte nuclear factors, forkhead box A, and GATA endodermal transcription factors in epithelial cancer cells. J Biol Chem 2007;282:22638-22650.

26. Renes IB, Verburg M, Van Nispen DJ, et al. Epithelial proliferation, cell death, and gene expression in experimental colitis: alterations in carbonic anhydrase I, mucin MUC2, and trefoil factor 3 expression. Int J Colorectal Dis 2002;17:317-326.

27. Engbaek K, Johansen KS, Jensen ME. A new technique for Gram staining paraffin-embedded tissue. J Clin Pathol 1979;32:187-190.

28. Rong M, Rossi EA, Zhang J, et al. Expression and localization of Muc4/ sialomucin complex (SMC) in the adult and developing rat intestine: implications for Muc4/SMC function. J Cell Physiol 2005;202:275-284.

29. Rossi EA, McNeer RR, Price-Schiavi SA, et al. Sialomucin complex, a heterodimeric glycoprotein complex. Expression as a soluble, secretable form in lactating mammary gland and colon. J Biol Chem 1996;271:33476-33485.

30. Motil KJ, Grand RJ. Growth failure in children and adolescents with inflammatory bowel disease. In: Tytgat GN, Bartelsman JFWM, van Deventer SJH (eds). Inflammatory Bowel Diseases. Kluwer Academic Publishers: Dordrecht, Booton, London, 1995, pp 588-597.

31. Levine A, Shamir R, Wine $E$, et al. TNF promoter polymorphisms and modulation of growth retardation and disease severity in pediatric Crohn's disease. Am J Gastroenterol 2005;100:1598-1604.

32. Sawczenko A, Azooz O, Paraszczuk J, et al. Intestinal inflammationinduced growth retardation acts through IL- 6 in rats and depends on the -174 IL-6 G/C polymorphism in children. Proc Natl Acad Sci USA 2005;102:13260-13265.

33. Knigge KL. Inflammatory bowel disease. Clin Cornerstone 2002;4: 49-60.

34. Idris N, Carraway KL. Sialomucin complex (Muc4) expression in the rat female reproductive tract. Biol Reprod 1999;61:1431-1438.

35. Renes IB, Verburg M, Van Nispen DJ, et al. Distinct epithelial responses in experimental colitis: implications for ion uptake and mucosal protection. Am J Physiol Gastrointest Liver Physiol 2002;283:G169G179.

36. de Koning BA, Lindenbergh-Kortleve DJ, Pieters R, et al. The effect of cytostatic drug treatment on intestine-specific transcription factors Cdx2, GATA-4 and HNF-1alpha in mice. Cancer Chemother Pharmacol 2006;57:801-810.

37. Hollingsworth MA, Swanson BJ. Mucins in cancer: protection and control of the cell surface. Nat Rev Cancer 2004;4:45-60.

38. Ho SB, Dvorak LA, Moor RE, et al. Cysteine-rich domains of muc3 intestinal mucin promote cell migration, inhibit apoptosis, and accelerate wound healing. Gastroenterology 2006;131:1501-1517.

39. Kawachi S, Jennings $S$, Panes J, et al. Cytokine and endothelial cell adhesion molecule expression in interleukin-10-deficient mice. Am J Physiol Gastrointest Liver Physiol 2000;278:G734-G743.

40. Ruiz PA, Shkoda A, Kim SC, et al. IL-10 gene-deficient mice lack TGFbeta/Smad signaling and fail to inhibit proinflammatory gene expression in intestinal epithelial cells after the colonization with colitogenic Enterococcus faecalis. J Immunol 2005;174:2990-2999.

41. Fuss IJ, Boirivant M, Lacy B, et al. The interrelated roles of TGF-beta and IL-10 in the regulation of experimental colitis. J Immunol 2002;168:900-908.

42. Fenton Jl, Hursting SD, Perkins SN, et al. Interleukin-6 production induced by leptin treatment promotes cell proliferation in an Apc (Min/+) colon epithelial cell line. Carcinogenesis 2006;27:1507-1515. 